\title{
Implicit Snakes: Active Constrained Implicit Models
}

\author{
T.S. Yoo ${ }^{1}$ and K.R. Subramanian ${ }^{2}$ \\ 1 National Library of Medicine, Natl. Inst. of Health, Bethesda, MD, 20894, USA \\ yoo@nlm.nih.gov \\ 2 Dept. of Computer Science, Univ. of North Carolina, Charlotte, NC, 28223, USA \\ krs@cs. uncc.edu
}

Extended Abstract: We are exploring new techniques for active contour models (popularly known as "snakes" [1) by combining active segmentation models with the constrained implicit surface model introduced by Savchenko, et al. [4] and reintroduced under the name of variational implicit surfaces as a modeling and shape morphing tool by Turk and O'Brien 5 . In related work, we pursued more efficient numerical methods for these techniques. For a more complete description of our modeling methods, see Morse 2. Here we show that an active surface model can be created by combining constrained implicit surfaces with a solver that minimizes an appropriate functional. In these examples, our energy minimization techniques are relatively simple; new work in combing the active implicit modeling techniques with aggressive energy functionals is in progress. By extracting implicit models from discrete volume data, we are diverging from similar modeling methods described by Yngve and Turk [6].
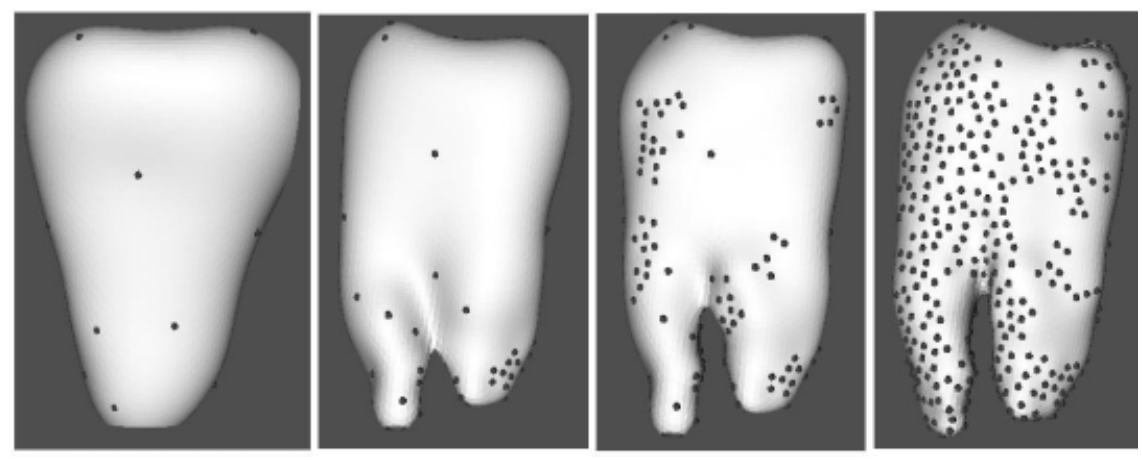

Fig. 1. Active interpolating implicit surfaces applied to a $256 \times 256 \times 163 \mathrm{CT}$ scan of a tooth. The algorithm was run for 21 iterations, incrementally adding constraints. The implicit model naturally suppresses sampling artifact, guaranteeing smoothness by the underlying radial basis interpolants. Constraints are indicated by small spheres on the surface boundary.

Figure 1 shows our method applied to an X-ray CT scan of a tooth. The first frame shows the first iteration of the progressive interpolating implicit surface 
algorithm. An initial set of constraints was actively moved toward areas of high boundary strength, and an interpolating implicit function was generated. The remaining frames show progressive refinement through 21 iterations, introducing additional constraints. Unlike a polygonal model, the final implicit surface can be sampled with arbitrary resolution. Figure 2 shows this process applied through a hybrid approach to intravascular ultrasound data (IVUS) of a canine aorta. The method correctly creates the partially occluded blood vessel from the slice data. The arterial vessel is segmented through a combination of $2 \mathrm{D}$ active contours and 3D interpolation. By definition, implicit surfaces are closely related to level

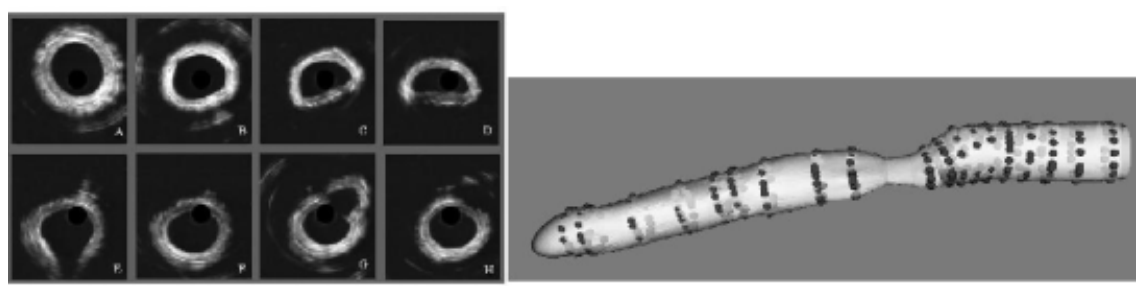

Fig. 2. Active constrained implicit surfaces generalized to active contours. The left panel shows ultrasound data collected using an endovascular transducer on a catheter. The data are slices, sampled at arbitrary angles along the vessel. Active interpolating surfaces were adapted to active interpolating contours to segment the individual slices. Particularly difficult slices due to poor signal can be avoided and the gaps interpolated if smoothness and connectedness can be assumed. Panel on the right shows the contours interpolated into an implicit surface, successfully mapping the $2.5 \mathrm{D}$ problem into the $3 \mathrm{D}$ domain. Crosssections of the resulting implicit surface can be used as priors to initialize active contour segmentation on interstitial slices

set techniques[3]. The advantages of implicit surfaces and level sets are their inherent ability to adapt to complex topologies. Our current focus is to employ in our methods improved partial differential equations similar to those used in level set algorithms.

\section{Acknowledgements}

We are indebted to Greg Turk, James O'Brien, and Gary Yngve for their insights and inspiration in implicit surfaces and to Bryan Morse, David Chen, and Penny Rheingans for their continued contributions to this and related work.

\section{References}

[1] M. Kass, A. Witkin, and D Terzopolous. Snakes: active contour models. Int. Journal of Computer Vision, 1:321-331, 1987. 
[2] B.S. Morse, T.S. Yoo, P. Rheingans, D.T. Chen, and K.R. Subramanian. Interpolating implicit surfaces from scattered surface data using compactly supported radial basis functions. In Proceedings of Shape Modeling International (SMI) 2001, pages 89-98, May 7-11, Genova, Italy, 2001.

[3] S. Osher and J. A. Sethian. Fronts propogating with curvature dependent speed: Algorithms based on hamilton-jacobi formulation. Journal of Computational Physics, 79:12-49, 1988.

[4] V. Savchenko, A. Pasko, G. Okunev, and T.L. Kunii. Fronts propogating with curvature dependent speed: Algorithms based on hamilton-jacobi formulation. Computer Graphics Forum, 14(4):181-188, 1995.

[5] G. Turk and J.F. O'Brien. Shape transformation using variational implicit functions. Computer Graphics Forum, 14(4):181-188, 1995.

[6] G. Yngve and G. Turk. Creating smooth implicit surfaces from polygonal meshes. Technical Report GIT-GVU-99-42, Graphics, Visualization and Usability Center, Georgia Institute of Technology, 1999. 